\title{
The bHLH transcription factor Hand2 regulates the expression of nanog in ANS
}

\section{differentiation}

Yusuke Hashimoto ${ }^{1}$, Reiko Myojin ${ }^{1}$, Naohiro Katoh ${ }^{1}$, Masaya Ohtsu ${ }^{1}$, Fumio Tashiro ${ }^{1}$, Fumitoshi Onoda ${ }^{1}$ and Yasufumi Murakami ${ }^{1}$

${ }^{1}$ Laboratory of Genome Biology, Department of Biological Science and Technology, Faculty of Industrial Science and Technology, Tokyo University of Science, 2641 Yamazaki, Noda, Chiba 278-8510, Japan

Correspondence to;

Dr. Yasufumi Murakami

Laboratory of Genome Biology

Department of Biological Science and Technology

Faculty of Industrial Science and Technology

Tokyo University of Science

2641 Yamazaki, Noda-shi, Chiba 278-8510, JAPAN

Tel: 81-471-24-1501 ex4408

FAX 81-471-22-1360

Email: yasufumi@rs.noda.tus.ac.jp 


\section{Abstract}

The basic helix-loop-helix transcription factor Hand2 is induced by bone morphogenetic proteins (BMPs) in neural crest-derived precursor cells during the early stage of development of the autonomic nervous system (ANS). Previous studies showed that Hand2 was essential for the ANS differentiation. However, regulatory mechanism of pluripotent genes has not been elucidated in ANS differentiation. Here, we show that Hand2 regulated nanog expression in ANS differentiation. Our studies demonstrated that the forced expression of Hand2 promoted the ANS differentiation program in P19 embryonal carcinoma (EC) cells without aggregation. Furthermore, our results suggested that Hand2 bound to the promoter of nanog, a gene required for embryonic stem cells self-renewal, and suppressed nanog expression after Hand2 induction. The rapid down-regulation of nanog mRNA during ANS differentiation correlated with the Hand2 transcriptional activity and nanog promoter methylation. These findings are evidence for a presence of the novel regulatory mechanism of nanog in ANS differentiation.

\section{Keywords}

Hand2, Nanog, P19 EC cells, autonomic nervous system 


\section{Introduction}

The development of sympathetic neurons is initiated by bone morphogenetic proteins (BMPs). BMPs control the expression of a group of transcription factors, including asc11, phox $2 b$, phox $2 a$, hand2, gata $2 / 3$ that play important roles in sympathetic neuron specification and differentiation $[1,2]$. However, it is not clarified how pluripotent genes are regulated in ANS differentiation.

The bHLH Hand2 (previously called dHand) is expressed in a number of tissues during development, including heart, limbs and crest-derived tissues, and has been shown to regulate the development of these tissues $[3,4,5]$. In the autonomic nervous system (ANS) of chicks and mice, Hand2 is expressed by sympathetic neurons, adrenal chromaffin cells and enteric neurons [6,7]. During development, hand2 expression is induced by BMPs but is first observed after the onset ascle1 and phox $2 b$ expression in chick sympathetic ganglion priomordia [8]. This finding, together with the lack of hand 2 expression in the phox $2 b$ knockout and maintained expression in the gata 3 knockout [9], implicates a function of hand 2 downstream of phox $2 b$ and upstream of gata2/3 in the group of BMP induced factors. Hand 2 overexpression was found to induce the generation of catecholaminergic neuron from neural crest precursor cells and P19 embryonal carcinoma cells [1,8], acting in concert with phox $2 b$, phox $2 a$ and ascll, which are ectopically expressed after hand 2 overexpression. Also upon forced phox $2 a$ expression, ascll and phox $2 b$ are induced, suggesting cross-regulation between these transcription factors, which are thus considered to be a network rather than a regulatory cascade $[10,11]$.

Nanog, a homeodomain protein exclusively expressed in embryonic stem cells (ESCs), is required to maintain self-renewal and the undifferentiated state of ESCs. nanog expression is rapidly down-regulated during ESC differentiation and the constitutive expression of nanog inhibits ESC differentiation[12,13].

To address the role of Hand 2 in ANS differentiation, we established that the exogenous expression of Hand 2 could be initiated by the withdrawal of doxycycline (Dox) in a P19-derived cell line. In the results, Hand2 expression activated the ANS differentiation program in P19-derived cell line. Furthermore, our data suggested that Hand2 bound to the promoter of nanog, a gene required for embryonic stem cells self-renewal, and suppressed nanog expression after Hand2 induction. Our studies revealed a novel mechanism in which Hand2 bound to the E-box in the promoter region of nanog which is one of a pluripotent master gene, and thereby promoted the ANS differentiation.

\section{Materials and Methods}

\section{Cell culture and construction of the Tet-controlled Hand2 expression system}

P19 EC cells were cultured in $\alpha$-MEM with 10\% fetal bovine serum (FBS; Japan Bioserum Co., Ltd., Nagoya, Japan), 100 $\mathrm{U} / \mathrm{ml}$ penicillin and $100 \mathrm{~g} / \mathrm{ml}$ streptomycin (Gibco). Tet-controlled Hand2 expression in the P19 cells was performed using the Tet-off ${ }^{\mathrm{TM}}$ gene expression system (Clontech). As a first step, the P19 cells were transfected with a pTet-Off vector using DOTAP and cultivated in the presence of $400 \mathrm{~g} / \mathrm{ml} \mathrm{G} 418$ (Wako, Tokyo, Japan) for selection. The resulting G418-resistant colonies were then screened by transient transfection with a pTRE2-Luc vector (Clontech) to isolate P19 Tet-off cells exhibiting a low background and high Tet-dependent induction of Luciferase by the withdrawal of Tet. The selected P19 Tet-off cell line was designated R13. At the second step, R13 cells were transfected with pTRE2-Hand2 and cultivated in the presence of $400 \mathrm{~g} / \mathrm{ml}$ hygromycin (Invitrogen) and $2 \mathrm{~g} / \mathrm{ml}$ doxycycline(Clontech) for selection. Hygromycin-resistant 
colonies were then screened for the induction of Hand 2 mRNA in the $\alpha$-MEM with $10 \%$ Tet system approved FCS (Clontech) by RT-PCR.

\section{Transient transfection}

P19 EC cells were maintained in $\alpha-$ MEM with $10 \%$ FCS. One day prior to transfection, the cells were plated in $10 \mathrm{~cm}$ dishes at a density of $4 \times 10^{6}$ cells per plate.

A total of $12 \mathrm{~g}$ /plate plasmid was transfected with P19 EC cells using Lipofectamine 2000 (Invitrogen, CA, USA). The cells were harvested after $48 \mathrm{~h}$.

\section{Bisulfate genomic sequencing}

Bisulfite treatment was performed using the EZ DNA Methylation-Gold Kit (Zymo Research, Orange, CA) according to the manufacturer's instructions. The PCR primers used to amplify nanog promoters are indicated in Supplementary Table 2. PCR products were cloned into pGEM-T easy vector (Promega, Madison, WI), and 11 randomly selected clones for each gene were sequenced with T7 forward and SP6 reverse primers.

\section{Immunocytochemistry}

Live cells were incubated with $5 \mathrm{~g} / \mathrm{ml}$ Hoechst 33258 (Molecular Probes, Eugene, OR) for $20 \mathrm{~min}$, rinsed in PBS, fixed in $100 \%$ ethanol for $10 \mathrm{~min}$ at $-20^{\circ} \mathrm{C}$, incubated in $100 \%$ acetone for $1 \mathrm{~min}$ at $-20^{\circ} \mathrm{C}$, and washed three times with PBS. The cells were incubated with blocking solution (10\% rabbit serum in PBS) for $1 \mathrm{~h}$ at room temperature, then incubated with primary antibody in 4\% rabbit serum in PBS for $1 \mathrm{~h}$. After washing, the cells were incubated in Alexa Fluor 488 conjugated anti-mouse and Alexa Fluor 594 conjugated anti-rabbit secondary antibodies (Invitrogen) for $30 \mathrm{~min}$. The antibodies were Peripherin (sc-7604; Santa Cruz Biotechnologies) and TuJ1 (MMS-435P; Covance, Berkeley, CA).

\section{nanog promoter construct and luciferase assays for promoter activity}

A 1.2-kb genomic DNA encompassing the promoter region of nanog was cloned into a promoterless luciferase construct, pGL3 Basic vector (Promega). Luciferase assays were performed using the Dual Luciferase system (Promega). Cells were plated at a density of $5 \times 10^{4}$ cells per well of a 24-well plate. nanog promoter luciferase constructs were transfected into the cells with a control vector by Lipofectamine according to the manufacturer's instructions (Invitrogen). 24 hours after transfection, the cells were replated in a 96-well plate with dox free medium. 24 hours after replating, the luciferase activities were analyzed using the Dual Luciferase system.

Western blot analysis, quantitive RT-PCR (qRT-PCR), ChIP assay were described in "Supplementary materials and methods"

\section{Results}

\section{Forced expression of Hand2 induced differentiation of PNS in P19 EC cells without aggregation}

To investigate whether the forced expression of Hand2 activated the peripheral nervous system (PNS) differentiation program, we established a cell line from P19 EC cells in which the exogenous expression of Hand2 could be initiated by the removal of Dox. Briefly, a pTRE2-Hand2 construct was transfected into the P19 EC cell line which harbored the pTet-Off 
vector (termed R13). The resulting stable transformants should have expressed Hand2 (termed P19-Hand2 cells) upon removal of Dox. To examine whether the expression of exogenous Hand 2 could be efficiently induced by the removal of Dox, we analyzed the expression level of the gene in the Tet-free medium. We detected the maximal protein expression of Hand2 after 48 h (Fig. 1A). However, the expression of Hand2 was not detected in P19 EC cells (data not shown). Using these stable transformants, we investigated whether Hand2 induces the differentiation of the PNS in P19 EC cells without aggregation by an immunocytochemical analysis (Fig. 1). In the presence of Dox (0 h), none of the transformants expressed either the pan-neuronal marker TuJ1 or the PNS marker peripherin (Figs. 1C and D). In contrast, when Hand2 was compulsorily expressed in the absence of Dox without aggregation, all transformants could differentiate into TuJ1 and peripherin-positive neurons at $96 \mathrm{~h}$ after removal of Dox, with an efficiency of above $80 \%$ (Fig. 1F-I and R). Neurons that use the same transmitter have many features in common, but they are operationally defined by the presence of the stored neurotransmitter. So, noradrenergic (NA) neurons are generally defined as neurons that express Th and Dbh [14]. Accordingly, we analyzed the expression of Th and Dbh after $72 \mathrm{~h}$ by immunocytochemistry stain analysis, and both noradrenergic neuron markers were detected (Fig. $1 \mathrm{~J}-\mathrm{Q}$ ). In conclusion, these neurons were determined to be NA neurons. These data suggested that the forced expression of Hand 2 induced the differentiation of the peripheral nervous system in P19 EC Cells.

\section{Forced expression of Hand2 activated the ANS differentiation program}

Previous studies have shown that the activation of the ANS differentiation program is accompanied by the activation of phox $2 a$ and phox $2 b$ as well as mash $1[8,15]$. To demonstrate that Hand2 induction activates the ANS differentiation program, we examined the changes in the expression level of the following genes during the PNS differentiation of P19 EC cells: phox2a, phox $2 b$, mash1, the noradrenergic markers $t h$ and $d b h$, nanog, the ectodermal marker otx2, the neural crest marker sox10 and the PNS marker prph1. Total RNAs were extracted from P19-Hand2 cells at various time points and were analyzed by qRT-PCR. Nanog is a recently discovered homeodomain transcription factor which is both sufficient and necessary for maintenance of the pluripotency of mouse ES cells and of mouse epiblasts [12,13]. nanog mRNAs decreased steeply after the induction of Hand2's expression (Fig. 2A), and otx2 and sox10 mRNAs increased after the induction of Hand2 (Figs. 2B and C); thereafter, the sox 10 mRNAs decreased to the basal level. The expression level of sox10 was changed in a manner that was antiparallel to that of prphl (Fig. 2D). phox 2b, mash1 and $d b h$ mRNA increased after the expression of Hand2 (Fig. 2E-G), phox $2 a$ mRNA increased after the expressions of phox $2 b$ and mash1 mRNA were induced (Fig. 2H). th mRNA was expressed, but not changed (data not shown). Taken together, these results indicate that, while the Hand2 expression inhibited genes necessary for the maintenance of pluripotency, it activated genes which are necessary for ANS differentiation. Thus, these results indicate that the forced expression of Hand 2 activated the ANS differentiation programs.

\section{Hand2 regulated the nanog transcription}

As nanog expression is immediately downregulated by the induction of Hand2 expression in ANS differentiation, we examined whether Hand 2 regulated expression of nanog. First, we examined the changes in the expression level of the following genes which were related to nanog expression during the ANS differentiation of P19 EC cells: $p 53$, tcf3, foxd3, oct4, and sox2. The tumor suppressor p53 can bind to the nanog promoter as suggested by both in vitro and in vivo evidence. During ES cell differentiation, the rapid downregulation of nanog correlates with the induction of the transcriptional activity [16]. Tcf3, a transcription factor that functions downstream of the Wnt pathway, is highly expressed in undifferentiated 
mouse ES cells. Tcf3 binds to a regulatory region on the nanog promoter and represses the promoter activity in ES cells [17]. FoxD3, a forkhead family transcription factor, is highly expressed in mouse ES cells and in pluripotent cells of the early embryo [18]. FoxD3 null mouse embryos die shortly after implantation with a loss of epiblast, a phenotype similar to Nanog deficient embryos, and FoxD3 is an activator of nanog [19,20]. The Oct4/Sox2 motif localized -180 bp upstream of the transcription start site was found to be important for nanog regulation. Recent studies have suggested that Oct4/Sox 2 acts to promote nanog transcription in pluripotent cells [21,22]. However, the expression level of these genes showed no change in ANS differentiation (Fig. 3A). Thus, we think that Hand2 regulates nanog transcription directly. To understand the molecular mechanism underlying Hand2-mediated suppression of nanog expression during ANS differentiation, we identified three putative Hand2-binding sites within the nanog promoter region (Fig. 3B). The first of these sites is denoted E1, and is located at -1255 to -1249 relative to the putative initiation ATG; the second site, denoted E2, is located at -975 to -969; and the third site, denoted E3, is located at -545 to -539 . We carried out a luciferase promoter assay as well as ChIP analysis of these Hand2-binding sites and bisulfate sequence analysis in order to examine the extent of methylation in the nanog promoter region. Moreover, we were not able to detect the recruitment of the co-activator CBP which is well known to increase transcription activity with Hand2 [23] by ChIP assay. nanog expression was significantly reduced in P19-Hand2 cells (Fig. 3C). Moreover, Hand2 and Dnmt3, which is the genomic DNA metyltransferase, bound to the nanog promoter region (Fig. 3D and Supplementary Figure 1). In addition, the nanog promoter [-265 to +15$]$ that was bound to the nanog transcription activator FoxD3 and Oct $4 /$ Sox 2 was 13\% methylated (Fig.3E), and the nanog first exon was hypermethylated after $48 \mathrm{~h}$ in P19-Hand2 cells (data not shown). These data suggested that Hand2 has the capability of regulating nanog transcription.

Previous studies have reported that nanog is located in the Wnt-signaling pathway [24,25]. In P19 EC cells, it has also been reported that the Wnt-signaling pathway is activated by aggregation [26]. Accordingly, we further analyzed whether Wnt1 could repress the nanog expression. The results suggested that neither Wnt1 activation by aggregation nor Wnt1 transient expression in P19 EC cells repressed nanog transcription (Fig. 3 F).

\section{Discussion}

Previous studies have shown that Hand2 overexpression itself could not activate the ANS neuronal developmental program in P19 EC cells, but could activate the development program if the transient expression was performed in conjunction with aggregation of the cells [15,27]. However, it seemed difficult to identify the target genes of Hand2 because the expression of numerous genes may well be altered by aggregation. To identify target genes for Hand2, we established a P19 derived cell line which exogenously expresses Hand2 upon removal of Dox. We also attempted to induce the differentiation of ANS in P19 EC cells without aggregation using this cell line, and succeeded in this attempt. On the other hand, as the transient Hand2 expression could not induce ANS differentiation, it suggests that the continuously elevated expression of Hand2 is required for ANS differentiation.

Our study showed that Hand2 induced ANS differentiation without aggregation. The role of the aggregation process in the differentiation has previously been discussed from various points of view. The neuronal differentiation of P19 EC cells is dependent on cell aggregation during the induction period [28]. Conditions that reduce adhesion in the aggregates inhibit the formation of neurons [29]. Although aggregation is clearly essential, little is known about the mechanism by which it promotes differentiation. Our present data suggested that Wnt-1, the vertebrate counterpart of the Drosophila wingless gene, is up-regulated by aggregation alone in P19 EC cells, as has been demonstrated previously [26]. Wnt-1 signals through a receptor complex composed of members of the Frizzled and low density lipoprotein families and 
activates the $\beta$-catenin/transcriptional T-cell factor signaling pathway (known as the canonical Wnt pathway) [30]. Pluripotent master genes, such as nanog, oct-3/4 and rex-1 are located at the downstream of the Wnt-signaling pathway $[24,25]$. Thus, we predicted that activation of Wnt signaling pathway suppressed the transcription of nanog in P19 EC cells. However, our results suggested Wntl activation alone is not sufficient for suppression of nanog transcription. Therefore, these data suggested that Hand2 regulated the transcription of nanog through another signaling pathway. In our study employing a ChIP assay and luciferase promoter assay, it was suggested that the downregulation of nanog by expression of Hand2 induced the recruitment of Dnmt 3 to the nanog promoter region. Consequently, the nanog promoter region, which is a binding site of the nanog transcription activators FoxD3 and Oct4/Sox2, was methylated by Hand2 induction. These data suggests that Hand2 regulates the nanog transcription by methylation of the nanog promoter in ANS differentiation. Our findings propose a novel regulatory mechanism of nanog transcription in ANS differentiation by Hand2. Previous studies have been focused on the genes which have a role to promote ANS differentiation. However, we found a novel regulatory mechanism of nanog, as follow, Hand2 repressed the nanog transcription by induction of methylation in nanog transcription activator sites with Dnmt3 during ANS differentiation. After the nanog repression, Hand2 activated the ANS-related genes. Finally, Hand2 induced the DBH, and P19EC cells differentiated into the ANS neurons (Fig. 3G).

It has already been shown that the exogenous expression of a couple of transcription factors converted fully differentiated mouse cells to iPS cells which can be differentiated to a variety of tissues [31]. This finding has stimulated the hunt for master gene(s) which regulate the differentiation of various stem cells. Our study has shown that the exogenous expression of one transcription factor (Hand2) activated the ANS differentiation program. Identification as well as analysis of the transcriptional regulators will be increasingly important for clarifying the molecular mechanism of such a differentiation program.

\section{Acknowledgements}

This work has been supported by the grants supplied by the Ministry of Education, Culture, Sports, Science and Technology (MEXT), the Japan Science and Technology Agency (JST), and Bio Matrix Research Inc.

\section{REFERENCES}

[1] M.J. Howard, Mechanisms and perspectives on differentiation of autonomic neurons, Dev Biol 277 (2005) 271-286.

[2] H. Rohrer, The role of bone morphogenetic proteins in sympathetic neuron development, Drug News Perspect 16 (2003) 589-596.

[3] D.G. McFadden, J. McAnally, J.A. Richardson, J. Charite, E.N. Olson, Misexpression of dHAND induces ectopic digits in the developing limb bud in the absence of direct DNA binding, Development 129 (2002) 3077-3088.

[4] D. Srivastava, T. Thomas, Q. Lin, M.L. Kirby, D. Brown, E.N. Olson, Regulation of cardiac mesodermal and neural crest development by the bHLH transcription factor, dHAND, Nat Genet 16 (1997) 154-160.

[5] H. Yanagisawa, D.E. Clouthier, J.A. Richardson, J. Charite, E.N. Olson, Targeted deletion of a branchial arch-specific enhancer reveals a role of dHAND in craniofacial development, Development 130 (2003) 1069-1078.

[6] B.A. Firulli, M.J. Howard, J.R. McDaid, L. McIlreavey, K.M. Dionne, V.E. Centonze, P. 
Cserjesi, D.M. Virshup, A.B. Firulli, PKA, PKC, and the protein phosphatase 2A influence HAND factor function: a mechanism for tissue-specific transcriptional regulation, Mol Cell 12 (2003) 1225-1237.

[7] M. Howard, D.N. Foster, P. Cserjesi, Expression of HAND gene products may be sufficient for the differentiation of avian neural crest-derived cells into catecholaminergic neurons in culture, Dev Biol 215 (1999) 62-77.

[8] M.J. Howard, M. Stanke, C. Schneider, X. Wu, H. Rohrer, The transcription factor dHAND is a downstream effector of BMPs in sympathetic neuron specification, Development 127 (2000) 4073-4081.

[9] K. Tsarovina, A. Pattyn, J. Stubbusch, F. Muller, J. van der Wees, C. Schneider, J.F. Brunet, H. Rohrer, Essential role of Gata transcription factors in sympathetic neuron development, Development 131 (2004) 4775-4786.

[10] M. Stanke, D. Junghans, M. Geissen, C. Goridis, U. Ernsberger, H. Rohrer, The Phox2 homeodomain proteins are sufficient to promote the development of sympathetic neurons, Development 126 (1999) 4087-4094.

[11] M. Stanke, J. Stubbusch, H. Rohrer, Interaction of Mash1 and Phox2b in sympathetic neuron development, Mol Cell Neurosci 25 (2004) 374-382.

[12] K. Mitsui, Y. Tokuzawa, H. Itoh, K. Segawa, M. Murakami, K. Takahashi, M. Maruyama, M. Maeda, S. Yamanaka, The homeoprotein Nanog is required for maintenance of pluripotency in mouse epiblast and ES cells, Cell 113 (2003) 631-642.

[13] I. Chambers, D. Colby, M. Robertson, J. Nichols, S. Lee, S. Tweedie, A. Smith, Functional expression cloning of Nanog, a pluripotency sustaining factor in embryonic stem cells, Cell 113 (2003) 643-655.

[14] C. Goridis, H. Rohrer, Specification of catecholaminergic and serotonergic neurons, Nat Rev Neurosci 3 (2002) 531-541.

[15] Y. Morikawa, Y.S. Dai, J. Hao, C. Bonin, S. Hwang, P. Cserjesi, The basic helix-loop-helix factor Hand 2 regulates autonomic nervous system development, Dev Dyn 234 (2005) 613-621.

[16] T. Lin, C. Chao, S. Saito, S.J. Mazur, M.E. Murphy, E. Appella, Y. Xu, p53 induces differentiation of mouse embryonic stem cells by suppressing Nanog expression, Nat Cell Biol 7 (2005) 165-171.

[17] L. Pereira, F. Yi, B.J. Merrill, Repression of Nanog gene transcription by Tcf3 limits embryonic stem cell self-renewal, Mol Cell Biol 26 (2006) 7479-7491.

[18] J. Sutton, R. Costa, M. Klug, L. Field, D. Xu, D.A. Largaespada, C.F. Fletcher, N.A. Jenkins, N.G. Copeland, M. Klemsz, R. Hromas, Genesis, a winged helix transcriptional repressor with expression restricted to embryonic stem cells, J Biol Chem 271 (1996) 23126-23133. 
[19] L.A. Hanna, R.K. Foreman, I.A. Tarasenko, D.S. Kessler, P.A. Labosky, Requirement for Foxd3 in maintaining pluripotent cells of the early mouse embryo, Genes Dev 16 (2002) 2650-2661.

[20] G. Pan, J. Li, Y. Zhou, H. Zheng, D. Pei, A negative feedback loop of transcription factors that controls stem cell pluripotency and self-renewal, FASEB J 20 (2006) 1730-1732.

[21] T. Kuroda, M. Tada, H. Kubota, H. Kimura, S.Y. Hatano, H. Suemori, N. Nakatsuji, T. Tada, Octamer and Sox elements are required for transcriptional cis regulation of Nanog gene expression, Mol Cell Biol 25 (2005) 2475-2485.

[22] D.J. Rodda, J.L. Chew, L.H. Lim, Y.H. Loh, B. Wang, H.H. Ng, P. Robson, Transcriptional regulation of nanog by OCT4 and SOX2, J Biol Chem 280 (2005) 24731-24737.

[23] Y.S. Dai, P. Cserjesi, The basic helix-loop-helix factor, HAND2, functions as a transcriptional activator by binding to E-boxes as a heterodimer, J Biol Chem 277 (2002) 12604-12612.

[24] H. Niwa, Molecular mechanism to maintain stem cell renewal of ES cells, Cell Struct Funct 26 (2001) 137-148.

[25] N. Sato, L. Meijer, L. Skaltsounis, P. Greengard, A.H. Brivanlou, Maintenance of pluripotency in human and mouse embryonic stem cells through activation of Wnt signaling by a pharmacological GSK-3-specific inhibitor, Nat Med 10 (2004) 55-63.

[26] S. Teramoto, F. Kihara-Negishi, T. Sakurai, T. Yamada, T. Hashimoto-Tamaoki, S. Tamura, S. Kohno, T. Oikawa, Classification of neural differentiation-associated genes in P19 embryonal carcinoma cells by their expression patterns induced after cell aggregation and/or retinoic acid treatment, Oncol Rep 14 (2005) 1231-1238.

[27] M.H. Farah, J.M. Olson, H.B. Sucic, R.I. Hume, S.J. Tapscott, D.L. Turner, Generation of neurons by transient expression of neural bHLH proteins in mammalian cells, Development 127 (2000) 693-702.

[28] E.M. Jones-Villeneuve, M.W. McBurney, K.A. Rogers, V.I. Kalnins, Retinoic acid induces embryonal carcinoma cells to differentiate into neurons and glial cells, J Cell Biol 94 (1982) 253-262.

[29] J.W. Schmidt, J.S. Brugge, W.J. Nelson, pp60src tyrosine kinase modulates P19 embryonal carcinoma cell fate by inhibiting neuronal but not epithelial differentiation, J Cell Biol 116 (1992) 1019-1033.

[30] Y. Hirabayashi, Y. Itoh, H. Tabata, K. Nakajima, T. Akiyama, N. Masuyama, Y. Gotoh, The Wnt/beta-catenin pathway directs neuronal differentiation of cortical neural precursor cells, Development 131 (2004) 2791-2801.

[31] K. Takahashi, S. Yamanaka, Induction of pluripotent stem cells from mouse embryonic and adult fibroblast cultures by defined factors, Cell 126 (2006) 663-676. 


\section{Figure legends}

Fig.1 Forced expression of Hand2 induced differentiation of NA neurons

The ability of Hand 2 to activate the neuronal program was examined by the forced expression of Hand2 without aggregation. (A) A P19-derived cell line in which the exogenous expression of Hand 2 could be initiated by the withdrawal of Dox was established. To examine whether the expression of exogenous Hand 2 could be induced by the removal of Dox, the time-dependent expression level of Hand2 was analyzed by Western blotting. (B-I): Cells were cultured for $96 \mathrm{~h}$ with Dox (B-E) or without Dox (F-Q) and analyzed for the expression of Tuj1 (pan-neuronal marker) or peripherin (peripheral neuronal marker). (B, F, J, N) Hoechst 33258, (C, G, K, O) Tuj1, (D, H) peripherin, (L) TH, (P) DBH, (E, I, M, Q) merge. (R): Ratio of Tuj1 (gray) or peripherin (white) -positive cells to Hoechst-labeled cells. Each value is the mean \pm S.E. of triplicate chamber slides; ${ }^{*} \mathrm{p}<0.0003,{ }^{* *} \mathrm{p}<0.00003$ compared with the P19-H2 cells cultured with Dox.

\section{Fig.2 Forced expression of Hand2 activated the ANS differentiation program}

(A-H) The expression levels of the genes that may be involved in ANS differentiation were characterized by qRT-PCR after induction of the Hand 2 gene expression. The quantities of mRNAs were determined by comparing the E11.5 embryo total RNA with a known amount of internal control RNA copies. (A) nanog, (B) otx2, (C) sox10, (D) prph1, (E) dbh, (F) mash1, (G) phox2b, (H) phox $2 a$. The expression level of beta actin mRNA was used as an internal control. Each value is the mean \pm S.E. of triplicate independent experiments in two clones.

\section{Fig.3 Hand2 regulated the nanog transcription}

(A) RT-PCR was performed to examine the expression of the genes regulating nanog transcription. Samples were collected at the indicated time after withdrawal of Dox. The relative ratios of the mRNA expression levels were estimated compared with the $0 \mathrm{~h}$ expression levels in each gene. The expression level of beta actin mRNA was used as an internal control. (B) nanog promoter region sequences. (C) At $24 \mathrm{~h}$ after withdrawal of Dox, the cells were lysed and assayed for luciferase activity. The relative luciferase activity of nanog-Luc compared with the promoterless vector is shown. Each value is the mean \pm S.E. of triplicate independent experiments; ${ }^{*} \mathrm{p}<0.0002,{ }^{*} \mathrm{p}<0.0007$. (D) ChIP analysis was performed with anti-Hand2 antibody and-CBP antibody or with Protein G Dynal magnetic beads as a negative control. (E) At $48 \mathrm{~h}$ after withdrawal of Dox, bisulfite genomic sequencing was performed to examine the DNA methylation profiles for individual CpG sites in the nanog promoter regions in P19 EC cells, R13 cells P19-Hand2 cells. The CpG dinucleotide locations are indicated. 11 clones were 
sequenced and are shown for each site. Open circles represent unmethylated cytosines, and closed circles represent methylated cytosines. (F) RT-PCR was performed to examine the expression of nanog or wht 1 mRNA in wild type P19 EC cells, pcDNA3.1-Wnt1 transfected P19 EC cells, or P19 EC cells with aggregation (Agg.) over days 0-2 or 0-4. (G)Model for the transcriptional cascade that regulates the NA neuron differentiation. At first, Hand2 expression is induced in P19 EC cells. This expression repressed the nanog transcription by induction of methylation in nanog transcription activator sites. On the other hand, Hand2 induction results in the independent activation of phox $2 b$ and mash 1 . The function of mash1 remains unclear, but it appears to promote the survival of neuronal progenitors and possibly also their differentiation. phox $2 b$ is required for the generation and maintenance of all noradrenergic cells. mash 1 and phox $2 b$ expression is required for phox $2 a$ and the genes encoding $\mathrm{TH}$ and $\mathrm{DBH}$ to be expressed. Thus, Hand2 induced the ANS differentiation by down-regulation of nanog transcription instead of aggregation and activated the ANS related genes. 
Figure.1

A

$0 h \quad 24 h \quad 48 h \quad 72 h$

$\mathrm{H}$ and2

G apdh

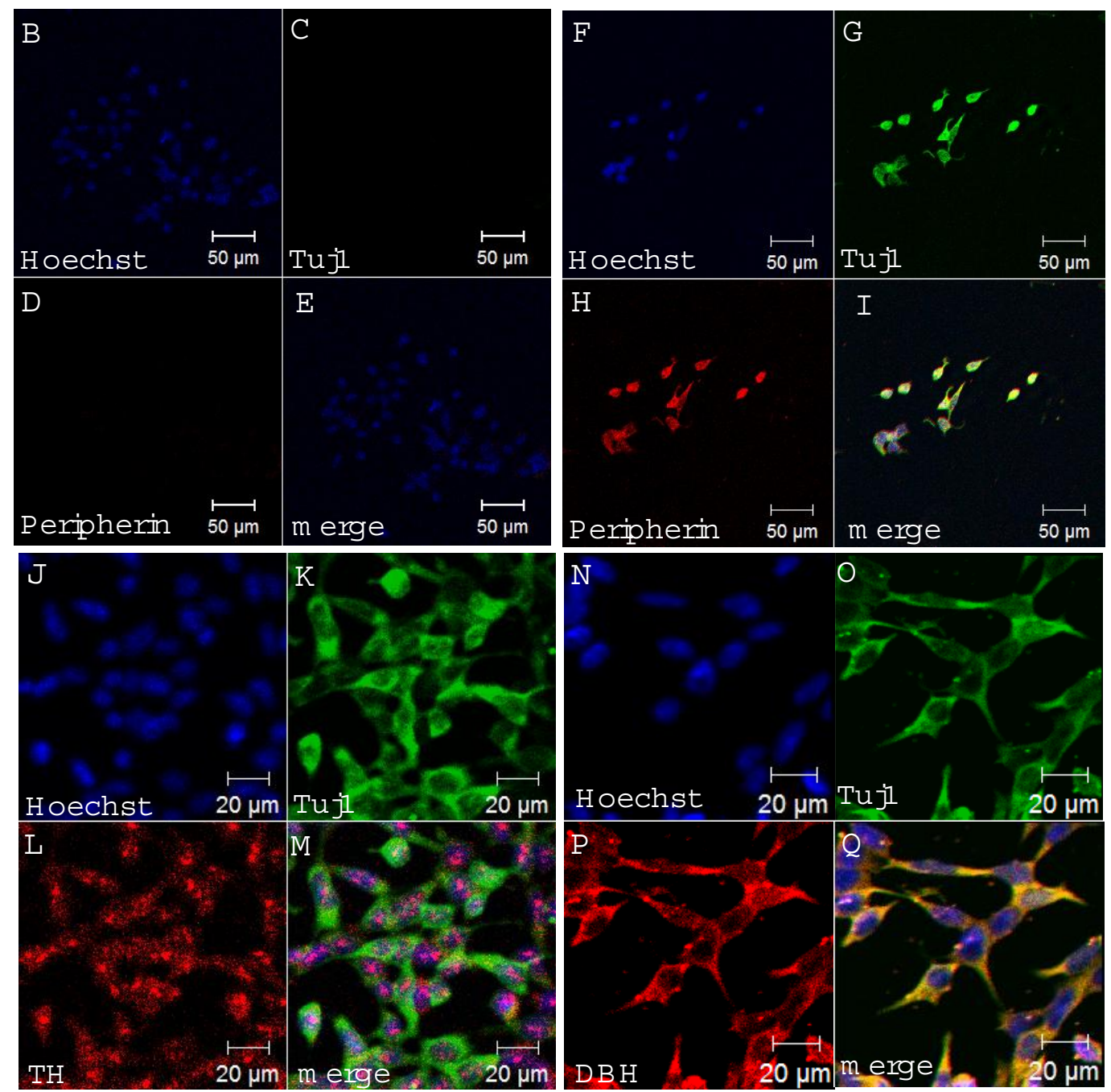

$\mathrm{R}$

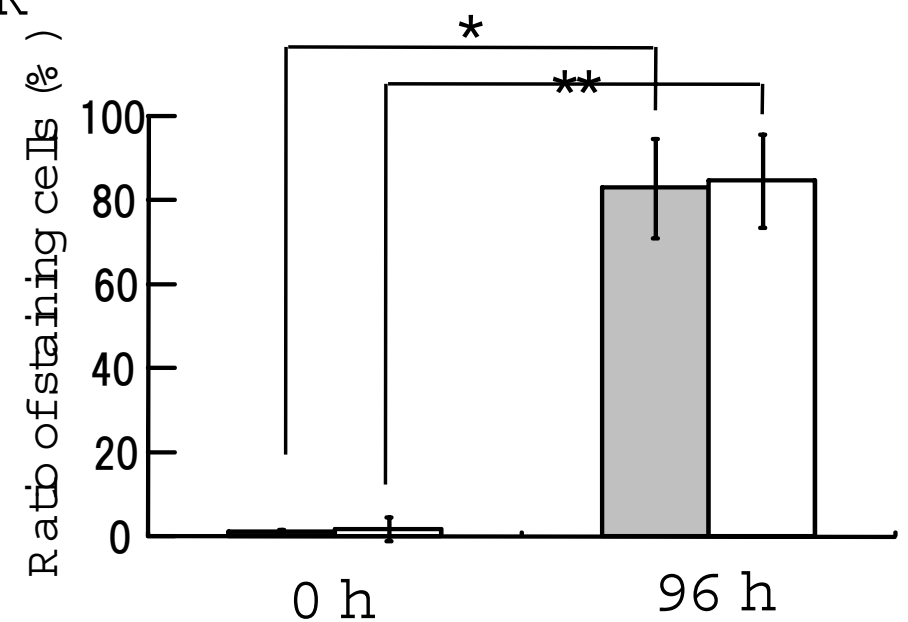


A Figure 2

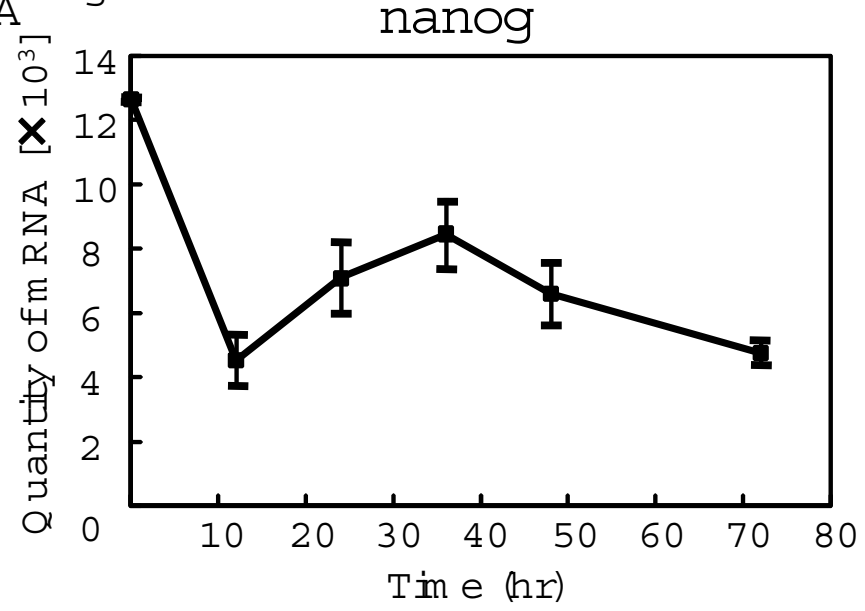

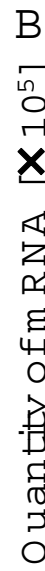

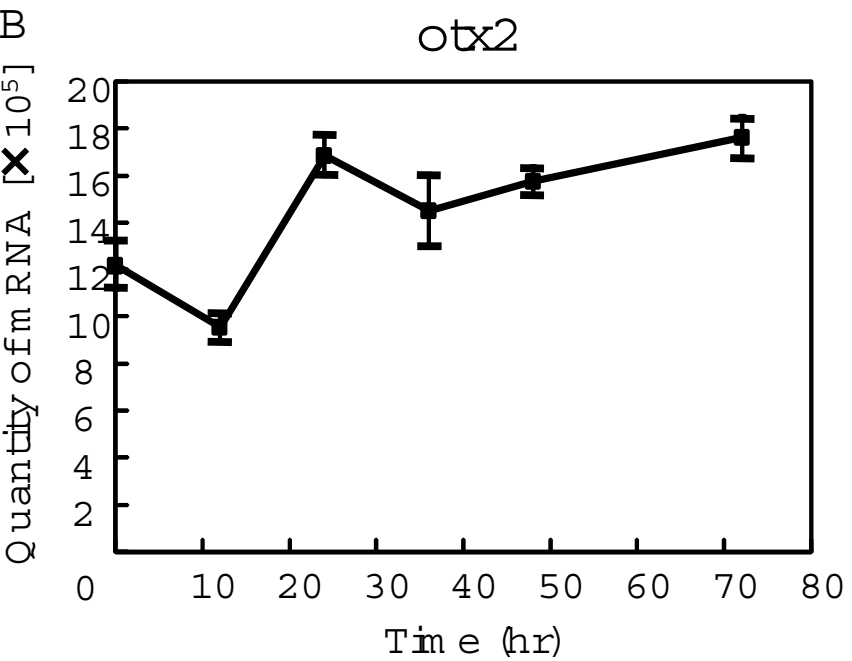

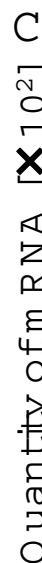

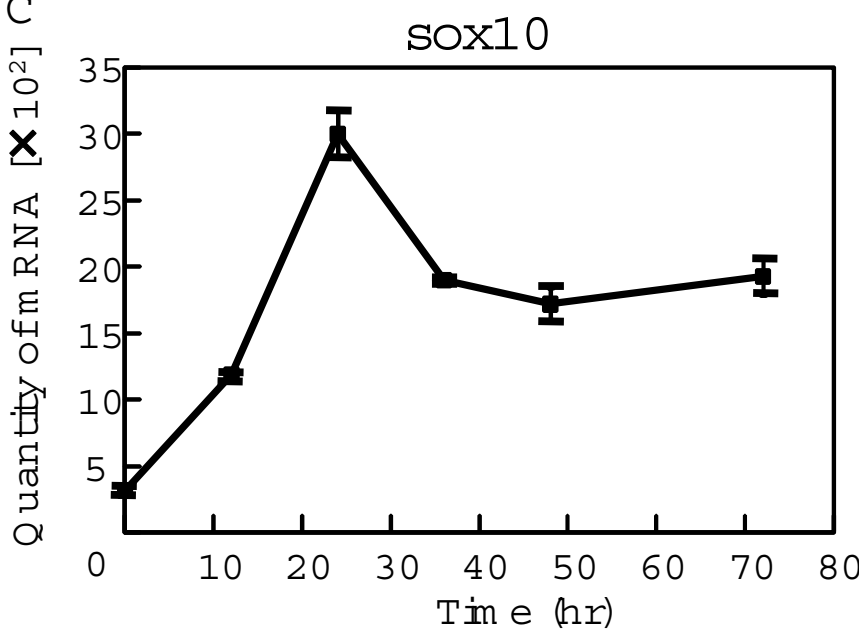

D

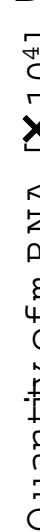

E

dbh

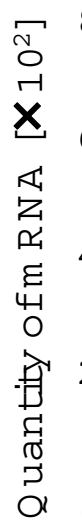

F

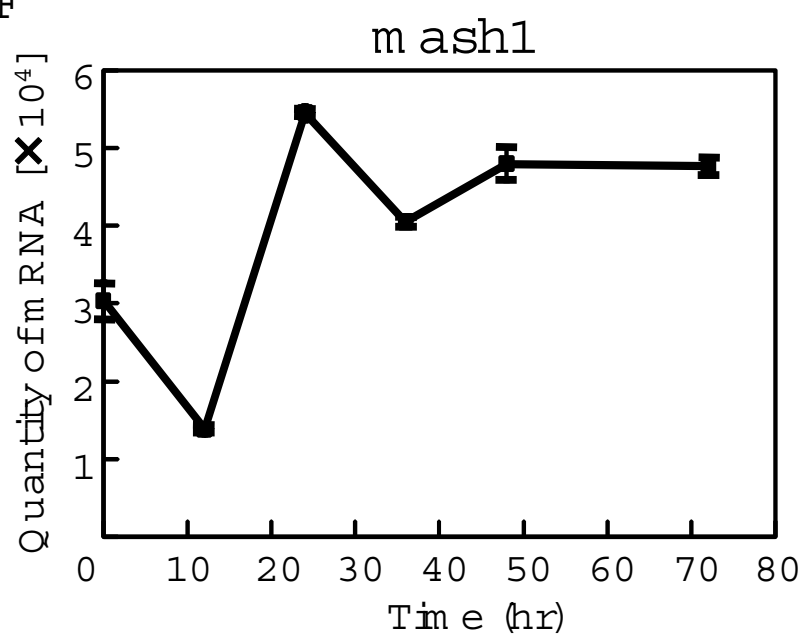

G

phox 2 b

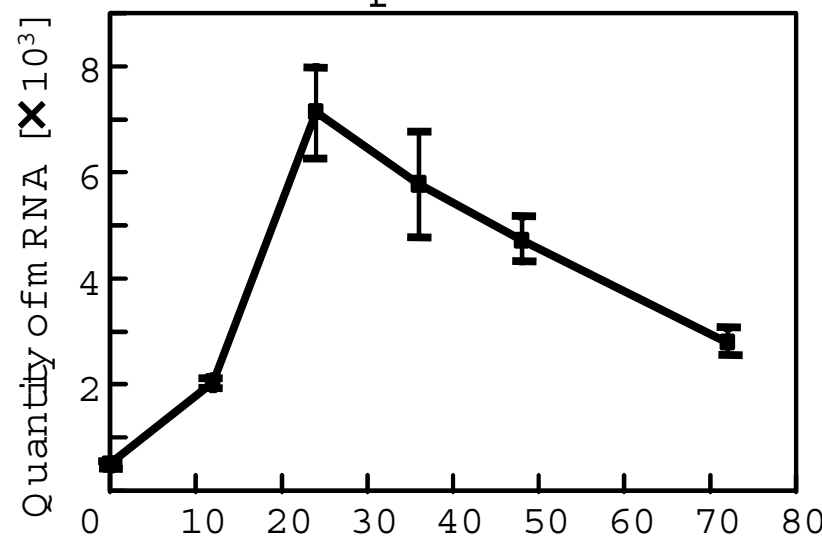

Tim e (hr)

$\mathrm{H}$

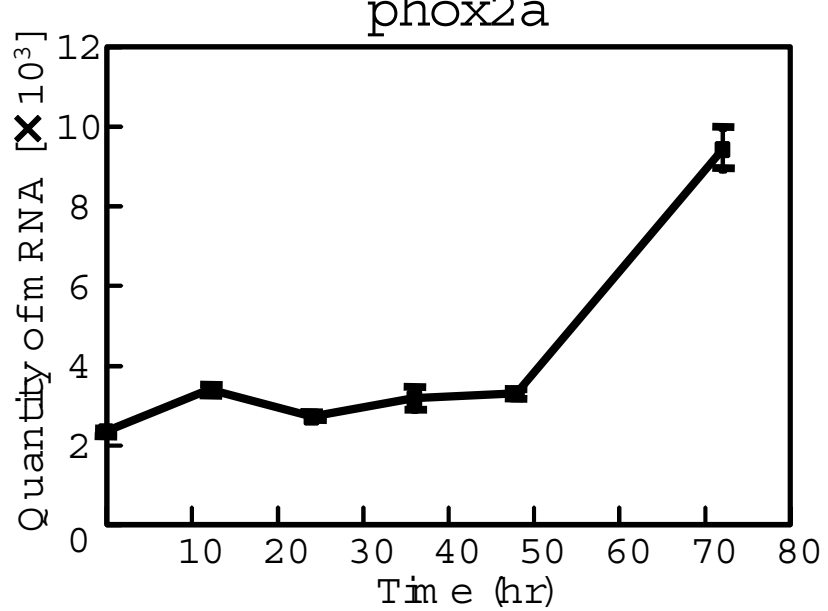


Figure 3

A

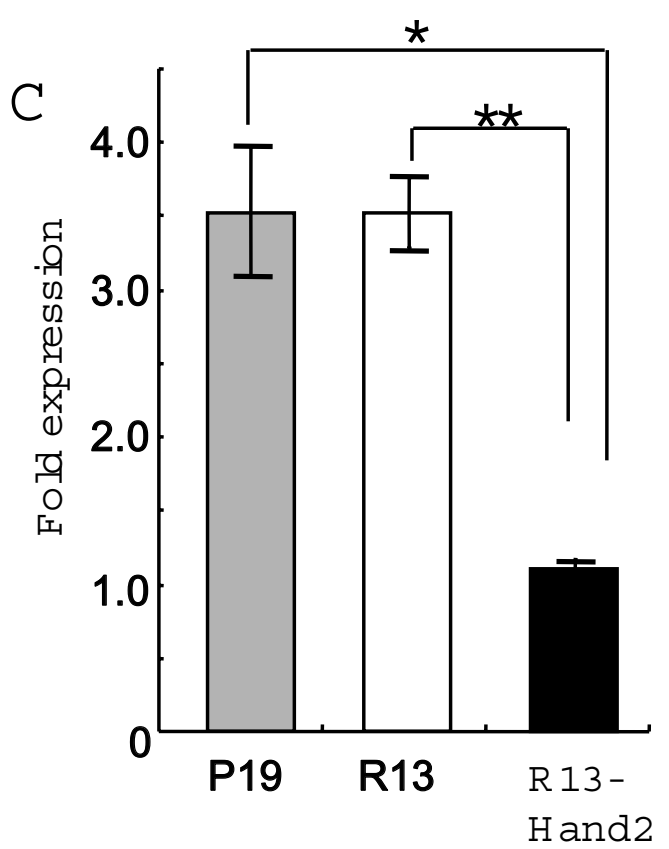

B

N anog prom oterregion

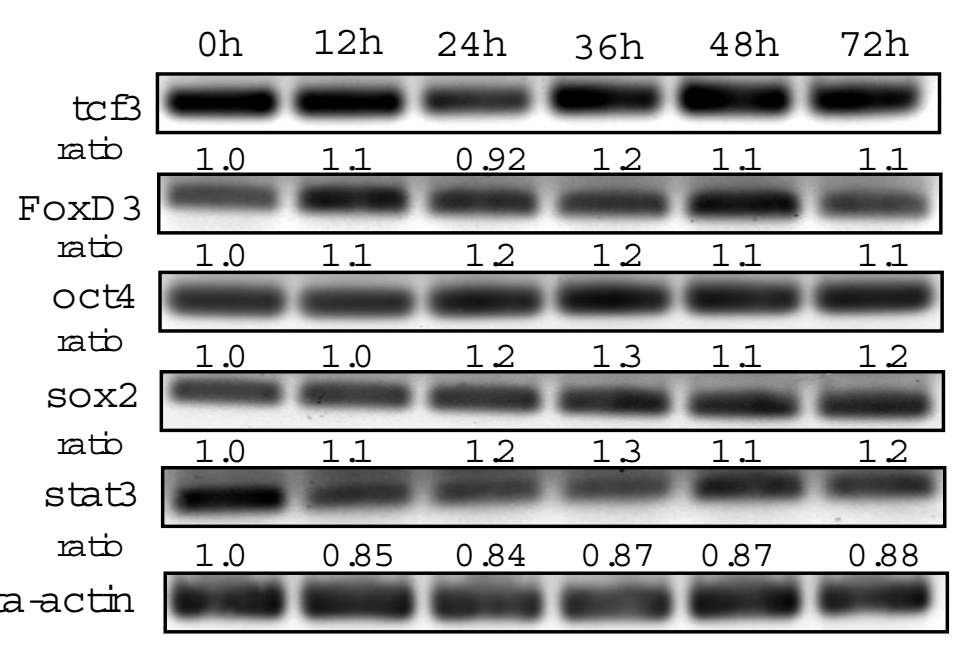

$\begin{array}{llllll}-1255 & -1249 & -975 & -969 & -545 & -539\end{array}$

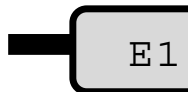

E2

E3

\section{Luciferase}

$\mathrm{E}$

P19-

pcDNA $3.1 \mathrm{H}$ and 2

pCDNA3.1

P 19

R 13

$\mathrm{H}$ and2

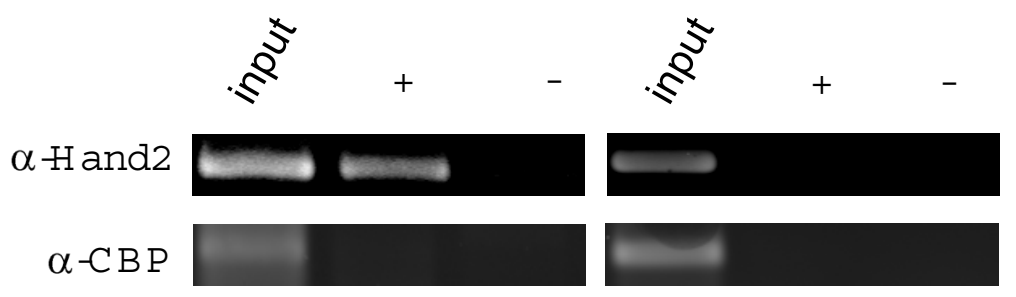
$\begin{array}{llllll}-265+15 & -265+15 & -265+15\end{array}$

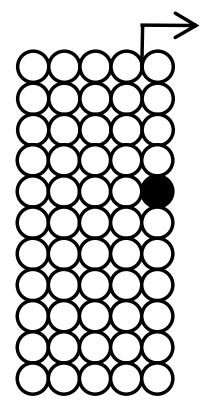

$0.01 \%$

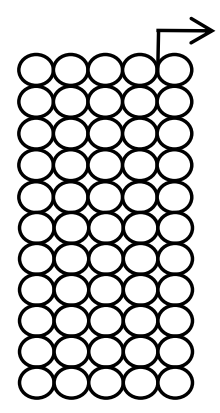

$0 \%$

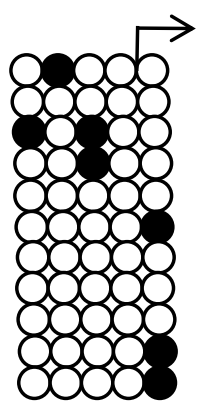

$13 \%$

G

F
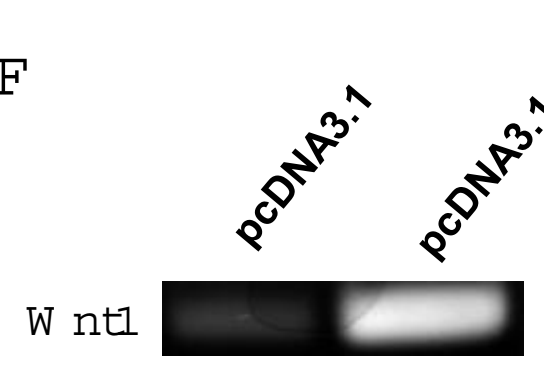

W ntl

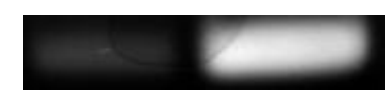

$\mathrm{N}$ anog

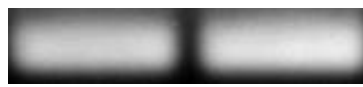

$\beta$-actin
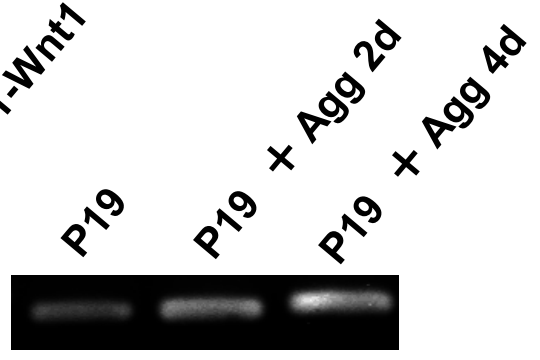

20

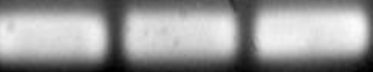

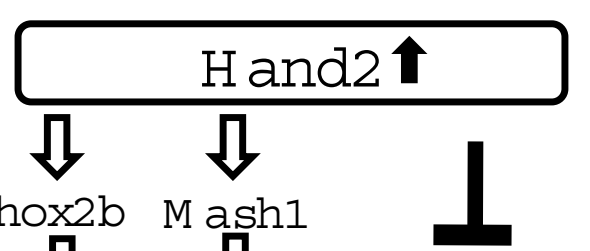

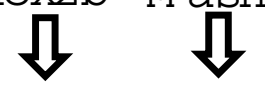

Phox2a

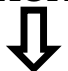

$\mathrm{TH}, \mathrm{DBH}$

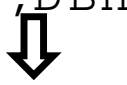

$\mathrm{N}$ anog

ANS N eurons 